\title{
The Pulsar Pair Plasma
}

\author{
Estelle Asseo \\ Centre de Physique Theorique, CNRS, Ecole Polytechnique, 91128 \\ Palaiseau, France \\ Alain Riazuelo \\ Département d'Astrophysique Relativiste et de Cosmologie, UMR 8629 \\ du CNRS, Observatoire de Paris, 92195 Meudon, France
}

\begin{abstract}
The anisotropic and relativistic features of the pulsar pair plasma are adequately modelled using relativistic one-dimensional JüttnerSynge distribution functions. The dispersion relation for wave propagation in such a plasma involves coefficients that specifically depend on the distribution function of its particles. An analytical determination of these coefficients allows us to obtain characteristics of quasi-longitudinal waves together with the conditions for the unstable interaction of ultrarelativistic beam and plasma. Similar derivations concern electromagnetic waves.
\end{abstract}

Pulsar pair plasmas are relativistic in two ways : because of the highly relativistic motion of electrons and positrons forming the pair plasma, and because of the relativistic temperatures of pair plasma particles. Besides, they are anisotropic because of the extreme strength of the pulsar magnetic field. The choice of adequate distribution functions to characterize flows of pair plasma particles in a pulsar emission region is extremely important, as the dispersion relation strongly depends on these very distribution functions :

$$
1+\sum_{\alpha}\left(\omega_{p \alpha}^{2} / k^{2}\right)\left(1 / n_{\alpha}\right) \int_{p_{\|}}\left(v_{\alpha}-v_{\|}\right)^{-1}\left(\partial f_{\alpha}\left(p_{\|}\right) / \partial p_{\|}\right) \mathrm{d} p_{\|}=k_{\perp}^{2} /\left(\omega^{2}-k_{\|}^{2}\right)
$$

Different distribution functions for each species $\alpha$ were considered in the literature, but relativistic generalization of Maxwellian distribution functions, that is relativistic one-dimensional Jüttner-Synge distribution functions, $f_{\alpha}\left(p_{\|}\right) \equiv$ $n_{\alpha} /\left[2 m K_{1}\left(\mu_{\alpha}\right)\right] \exp \left[-\mu_{\alpha} \gamma\left(p_{\|}\right)\right]$, are the most natural choice [with temperature parameter $\mu_{\alpha} \equiv m / k_{B} T_{\alpha}$, modified Bessel function $K_{1}\left(\mu_{\alpha}\right)$ and Lorentz factor associated with the flow $\left.\gamma\left(p_{\|}\right)\right]$. From this, we have obtained analytical expressions of dispersion relations in different limits (Asseo \& Riazuelo 1999). For instance, as long as the phase velocity is much larger than the thermal velocity, $v_{\phi} \gg v_{\text {th }}$, the dispersion relation for quasi-longitudinal waves is a sum of terms with coefficients $\zeta_{1}, \eta_{1}, \xi_{1}, \ldots$ namely :

$1-\sum_{\alpha}\left(\omega_{p \alpha}^{2} / k_{\|}^{2}\right)\left(v_{\phi}-v_{\alpha}\right)^{-2}\left[\zeta_{1}+\eta_{1} /\left(v_{\phi}-v_{\alpha}\right)+\xi_{1} /\left(v_{\phi}-v_{\alpha}\right)^{2}+\cdots\right]=k_{\perp}^{2} /\left(\omega^{2}-k_{\|}^{2}\right)$.

These coefficients depend on the temperatures, velocities and Lorentz factors of the flows. For the ultra-relativistic pair plasma, $\mu_{\alpha} \ll 3$, they are proportional 
to the temperature parameter $\mu_{\alpha}$ :

$$
\zeta_{1}^{R}=\mu_{\alpha}-\frac{\pi}{2} \mu_{\alpha}^{2} \gamma_{\alpha}+\mu_{\alpha}^{3}\left[1+2 v_{\alpha}^{2}+\mathcal{O}\left(v_{\alpha}^{4}\right)-\gamma_{\alpha}^{2}\left[\gamma_{E}+\ln \left(\mu_{\alpha} / 2\right)\right]\right]+\mathcal{O}\left(\mu_{\alpha}^{4}\right),
$$

together with similar expressions for $\eta_{1}^{R}$ and $\xi_{1}^{R}$ ( $\gamma_{E}$ is Euler's constant).

Thus in the observer's frame, for the interaction between ultra-relativistic beam and pair plasma, the frequency of resonant "Langmuir" waves, $\omega \simeq$ $\sqrt{\gamma_{p \alpha}} \omega_{p \alpha} / \sqrt{\mu_{\alpha}}$, is enhanced by a factor $1 / \sqrt{\mu_{\alpha}}$ relatively to its value in the weakly relativistic case. Considering the interaction between the primary beam and secondary pair plasma present in a pulsar emission region, pulsar parameters imply a wave frequency out of the radio domain : resonant unstable "Langmuir" waves are probably not responsible for radio events, in agreement with Cheng \& Ruderman (1977) conclusions and Melrose \& Gedalin (1999) analysis.

Otherwise, for the interaction between drifting electrons and positrons that form the ultra-relativistic pair plasma itself, it is not possible to model onedimensional Jüttner-Synge distribution functions, and to extend our analytical results. However, numerical analysis shows an efficient two-stream instability to be raised up in this case (Asseo \& Melikidze 1998).

A similar analysis is possible for electromagnetic transverse waves. In the strong pulsar magnetic field, it is only for oblique propagation relatively to the magnetic field direction that frequencies are modified by temperature effects. For instance, assuming an ultra-relativistic pair plasma, perpendicular wavevector and magnetic field, and a phase velocity much larger than unity, $v_{\phi} \gg 1$, Magneville (1990) obtains for the dispersion relation, using a three-dimensional Jüttner-Synge distribution function :

$$
\omega^{2} \simeq k^{2}+\omega_{p}^{2}(\mu / 3)\left[1-\left(\mu^{2} / 2\right)+\left(k^{2} / \omega^{2}\right)\left(1-\mu^{2}\right) / 5\right] .
$$

The one-dimensional case has only slightly different numerical coefficients. Thus, wave propagation in the ultra-relativistic pair plasma essentially arise at a frequency $\omega \propto \omega_{p} \sqrt{\mu}$. As the temperature parameter $\mu$ should be very small (Daugherty \& Harding 1983), the wave is a radio wave in the region of dominant magnetic field close to the surface of the star. The generation mechanism for such a kind of transverse electromagnetic waves, and the possibility that they reach an observer, remain as open problems.

As a result of our linear treatment, we conclude that in a pulsar emission region, relativistic and anisotropic features of pulsar pair plasma flows are essential for the interpretation of observed radio radiation from pulsars.

\section{References}

Asseo, E., Riazuelo, A. 1999, MNRAS submitted

Asseo, E., Melikidze, G.I. 1998 MNRAS, 301, 59

Cheng, A.F., Ruderman M.A. 1977, ApJ, 212, 800

Daugherty, J.K., Harding A.K. 1983, ApJ, 273, 761

Magneville, A. 1990, J. Plasma Physics, 44, 191; 44 (1990) 213

Melrose, D.B., Gedalin, M.E. 1999, ApJ submitted (preprint) 